\title{
edmetic
}

Revista de Educación Mediática y TIC

Las TIC y la educación social en el siglo XXI

ICT and social education in the twenty-first century

Fecha de recepción: 10/07/2015

Fecha de revisión: 30/10/2015

Fecha de aceptación: 09/12/2015 


\section{Las Tic y la educación social en el siglo XXI}

\section{ICT and social education in the twenty - first century}

\section{Begoña E. Sampedro Requena'}

\section{Resumen:}

Este artículo recoge una serie de reflexiones sobre el papel que juega los profesionales de la educación social en las nuevas realidades sociales, donde subyace la necesidad de una formación y capacitación de los recursos tecnológicos como elementos que favorecen la integración de los sujetos en la sociedad.

Como resultado de esta premisa se presenta un breve análisis teórico de la educación social en el siglo XXI, deteniéndonos en dos elementos esenciales de la misma, la titulación universitaria en la que se desarrolla y las características básicas de la ocupación profesional que la desempeñan.

Asimismo, se relata los elementos que determinan las sociedades actuales, aproximándonos a las tecnologías como componente básico de estas nuevas realidades.

\section{Palabras clave:}

Educación social; TIC; socialización; competencia digital.

\section{Abstract:}

This article contains a series of thoughts on the role played by professionals of social education in new social realities, in which underlies the necessity of a formation and training of technological resources as elements that promote integration of subjects in community. As a result this assumption, presents a brief theoretical analysis social education in the XXI century stopping at two main elements, university degree that develops and basic characteristics of

\footnotetext{
${ }^{1}$ Universidad de Córdoba. bsampedro@uco.es
} 
professional occupation which perform .

Also, is recounted the elements that determine today's societies, approaching technology as a basic component of these new realities

\section{Keywords:}

Social education; ICT; socialization; digital competence 


\section{1.- Introducción}

La práctica de la Educación Social se ha desarrollado en toda Europa con anterioridad al constructo de su formación, donde las titulaciones inherentes a esta ocupación laboral son relativamente más jóvenes.

Esta circunstancia es debida a su aplicabilidad en la sociedad actual, al remontarnos al origen etimológico, de la conjunción de términos, que la determina, nos encontramos con la expresión "educación", la cual según el Diccionario de la Real Academia Española (en adelante DRAE) en su $22^{a}$ edición (2001 y con enmiendas del 2012) presenta entre sus acepciones la acción y el efecto de educar; la crianza, enseñanza y doctrina que se da a los niños y jóvenes; e, instrucción por medio de la acción docente. Por otra parte, al detenernos sobre el otro vocablo que articula este término "social" el DRAE (2001 y con enmiendas del 2012) nos indica que su significado primordial es la pertenencia o aspecto relativo a la sociedad. Lo cual sugiere la extensión y diversidad de la expresión educación social, al considerar que se adhiere a dos campos epistemológicos: el ámbito de las ciencias de la educación y el ámbito de la sociología.

Esta unión de conjuntos multidisciplinares puede resultar cíclica y reiterativa, incluso compleja, dado que las demandas sociales transforman el espectro educativo $y$, que el mismo se devuelve para beneficio de la sociedad, mediante el desarrollo de las potencialidades y las capacidades del alumnado, que termina con la integración, relación y actuación social.

Por consiguiente, el educador social se convierte en un agente que potencia y facilita la adaptación del individuo a la sociedad, favoreciendo su socialización; y es precisamente, en la actualidad, donde este profesional adquiere mayor relevancia, dado que las tecnologías influyen en la interacciones con los demás; en la incorporación activa como ciudadanos; en el desenvolvimiento en la sociedad; y en el propio desarrollo comunitario y social. 


\section{La educación Social en el siglo XXI}

En la titulación de Educación Social confluyen muchas disciplinas, las cuales aportan sus propias concepciones y teorías al concepto, aunque existen unos elementos comunes y generalizables al mismo, los cuales como indica Pérez Serrano (2005: 9-10) son:

- "Debe buscar siempre el cambio social.

- Socializa y permite la adaptación social.

- Trabaja fundamentalmente acerca de problemas humanosociales.

- Tiene lugar, habitualmente, en contextos no formales.

- Tiene un campo de acción importante en la marginación, pero su radio de acción no se limita a ese ámbito.

- Exige estar en permanente contacto con la realidad.

- Requiere una intervención cualificada.

- Puede y debe ser aplicada en contextos escolares.

- Trata de hacer protagonista de su cambio a los propios destinatarios".

Estos aspectos intrínsecos a la Educación Social, ponen de relieve su finalidad elemental la socialización de los individuos, procurando la adaptabilidad y el ejercicio social mediante la intervención educativa en todos los contextos (formales, informales y no formales) donde se involucre estos.

Precisamente, la dificultad o complejidad en el análisis de la Educación Social proviene de su aplicabilidad, dado que cada sociedad tiene su propia organización social, al igual que cada contexto posee su peculiaridad; a consecuencia, en Europa, existe más de una titulación que proyectaría ocupaciones encaminadas a la socialización de los individuos. 


\subsection{El Grado de Educación Social}

Los países que conforman la Unión Europea ofrecen la titulación de grado de Educación Social con diversas denominaciones, desarrollando distintos conocimientos y aprendizajes relacionados con la intervención social y comunitaria; sin embargo todos los itinerarios ofertados consideran las exigencias que demandan el sector laboral para su ejercicio en la sociedad actual.

"La figura de la Educación Social en España es producto de tres históricas ocupaciones diferentes que son los educadores especializados, los animadores socioculturales y los educadores de adultos, en cuyos ejes, existen puntos de encuentro como la inadaptación, analfabetización, exclusión, marginación social, integración, capacitación profesional, desarrollo comunitario, etc." (Chamseddine, 2013:3-4).

En cuanto, a la estructura del Grado de Educación Social, el Espacio de Europeo de Educación Superior, efectuó una homogeneización de todas las titulaciones para los países miembros, organizando estos estudios en módulos genéricos y específicos. La tabla 1, muestra los módulos genéricos y su descripción para este grado universitario.

Tabla 1: Módulos genéricos del Grado de Educador Social.

Fuente: Elaboración propia a partir de las aportaciones de la ANECA (2005:164).

\section{MÓDULOS DESCRIPCIÓN}

Bases conceptuales y contextuales Fundamentos filosóficos, teóricos, de la educación antropológicos, históricos, comparados, biológicos, psicológicos, sociales, culturales, económicos, políticos, etc. Dimensión ética y estética, dimensión europea de la educación, bases de la interculturalidad

Intervención socioeducativa en Metodologías, proceso de enseñanza contextos no formales e informales aprendizaje y evaluación en contextos no formales - informales

Metodologías de la investigación en Medición y desarrollo de instrumentos, 
la intervención socioeducativa y investigación-acción, aplicación de tecnologías educativas las TIC

Diseño, gestión y evaluación de Diseño, gestión, desarrollo, y programas de intervención evaluación de proyectos, programas y socioeducativa acciones, socioeducativas

Técnicas, medios y recursos en la Aplicación de técnicas, medios y intervención socioeducativa recursos en los diversos ámbitos de intervención socioeducativa

Prócticum Prácticas en entornos relacionados con los ámbitos de trabajo de este agente.

Estos módulos genéricos constituyen los aspectos formativos elementales que proporcionan al alumnado que cursa el Grado de Educación Social de las herramientas cognitivas, procedimentales y actitudinales necesarias para desarrollar su futura labor profesional.

\subsection{La ocupación laboral de Educación Social}

En la Clasificación Internacional Uniforme de Ocupaciones (CIUO - 08), donde se recopilan las ocupaciones reguladas en toda Europa, no aparece de forma explícita la Educación Social; en cambio sí se presentan los profesionales del Trabajo Social. En concreto, en España, existe una Resolución de 5 de junio de 2007, que recoge el primer convenio colectivo con marco estatal de acción e intervención social, en el cual encajaría las ocupaciones relativas a esta titulación que venimos analizando.

Por Acción e Intervención Social, se entienden las actividades o acciones, que se realizan de manera formal $u$ organizada, que responden a necesidades sociales, que su propósito puede ser tanto prevenir, paliar 0 corregir procesos de exclusión social, como promover procesos de inclusión o participación social (Art. 2.2. de la Resolución de 5 de junio de 2007).

Esta definición de la ocupación laboral para el campo de la intervención social se ajusta a la formación universitaria que reciben los estudiantes del Grado de Educación Social, el cual desarrolla competencias 
genéricas o transversales de tipo instrumental, interpersonal y sistémicas como indica el Proyecto Tuning (González y Wagenaar, 2003) para el ejercicio de esta profesión descrita.

Aunque, sigue existiendo complejidad en la atribución de funciones y cualificación de estos profesionales a nivel europeo; sin embargo, la Asociación Estatal de Educación Social y, por tanto el Consejo General de Colegios de Educadores y Educadoras Sociales determinan seis funciones propias para la ocupación de estos profesionales:

- "Transmisión formación, desarrollo y promoción de la cultura

- Generación de redes sociales contextos, procesos y recursos educativos y sociales

- Mediación social, cultural y educativa.

- Conocimiento, análisis e investigación de los contextos sociales y educativos.

- Diseño, implementación y evaluación de programas y proyectos en cualquier contexto educativo.

- Gestión, dirección, coordinación y organización de instituciones y recursos educativos" (Asociación Estatal de Educación Social, 2007:46-47).

Estas seis funciones guardan coincidencia con los módulos genéricos de formación para los títulos universitarios de Educación Social, anteriormente expuestos; de donde se infiere que esta formación académica se orienta a capacitar al estudiante para trabajar en el ámbito educativo, social y cultural.

Al analizar la segunda, la tercera y la curta función que se le atribuye al profesional de la Educación Social, se puede reflexionar que las mismas se encaminan directamente a la intervención en contextos educativos (formales, informales y no formales); concretamente en aspectos relativos a la interacción e inclusión de los individuos en la educación.

En conclusión, como señala Gairín (2004), la transmisión de la educación y la cultura a las personas que integran una sociedad se realiza a través del 
sistema educativo, el cual posibilita la socialización en un determinado contexto; esta finalidad constituye la labor principal de los agentes de la Educación Social, que encuentran en los centros educativos un espacio sólido para su desenvolvimiento profesional.

\section{Efectos de las TIC en las sociedades del siglo XXI}

En los últimos años, todas las esferas de la ciudadanía se han visto modificadas por las transformaciones tecnológicas acaecidas; un ejemplo lo encontramos en el ámbito laboral, las formas de entender las ocupaciones se han visto afectadas por las formas de producción, dado que las mismas se han tecnificado originando nuevas concepciones profesionales. Asimismo, aumenta la tendencia a gestionar, tramitar y consultar de forma electrónica todos los aspectos relativos a la administración o burocratización; los mass media reclaman la participación de la audiencia a la que se dirigen enfatizando la interacción a través de las redes sociales. Aunque, no sólo estos ámbitos se han visto afectados sino también la esfera educativa se ha visto alterada, reformulando las normativas, los currículos, las metodologías y los procesos de enseñanza - aprendizaje, como señala Castells (2005:3), "se puede argumentar, que hoy en día, la riqueza, el poder, y la generación de conocimiento depende en gran medida de la capacidad de organizar la sociedad para aprovechar los beneficios del nuevo sistema tecnológico, (...). Entiendo que la conceptualización de la sociedad en red es como la estructura social resultante de la interacción entre el nuevo paradigma tecnológico y la organización social en genera"l.

Las tecnologías, de carácter comunicativo e informacional, como indican Martín, López y González (2013:1) "han tenido un desarrollo explosivo en la última parte del siglo XX y el comienzo del siglo XXI, al punto de que han dado forma a lo que se denomina Sociedad del Conocimiento o de la Información". 
En este sentido Martínez Sánchez (2007) expone un conjunto de características que definen la organización social que se origina de esta nueva ciudadanía.

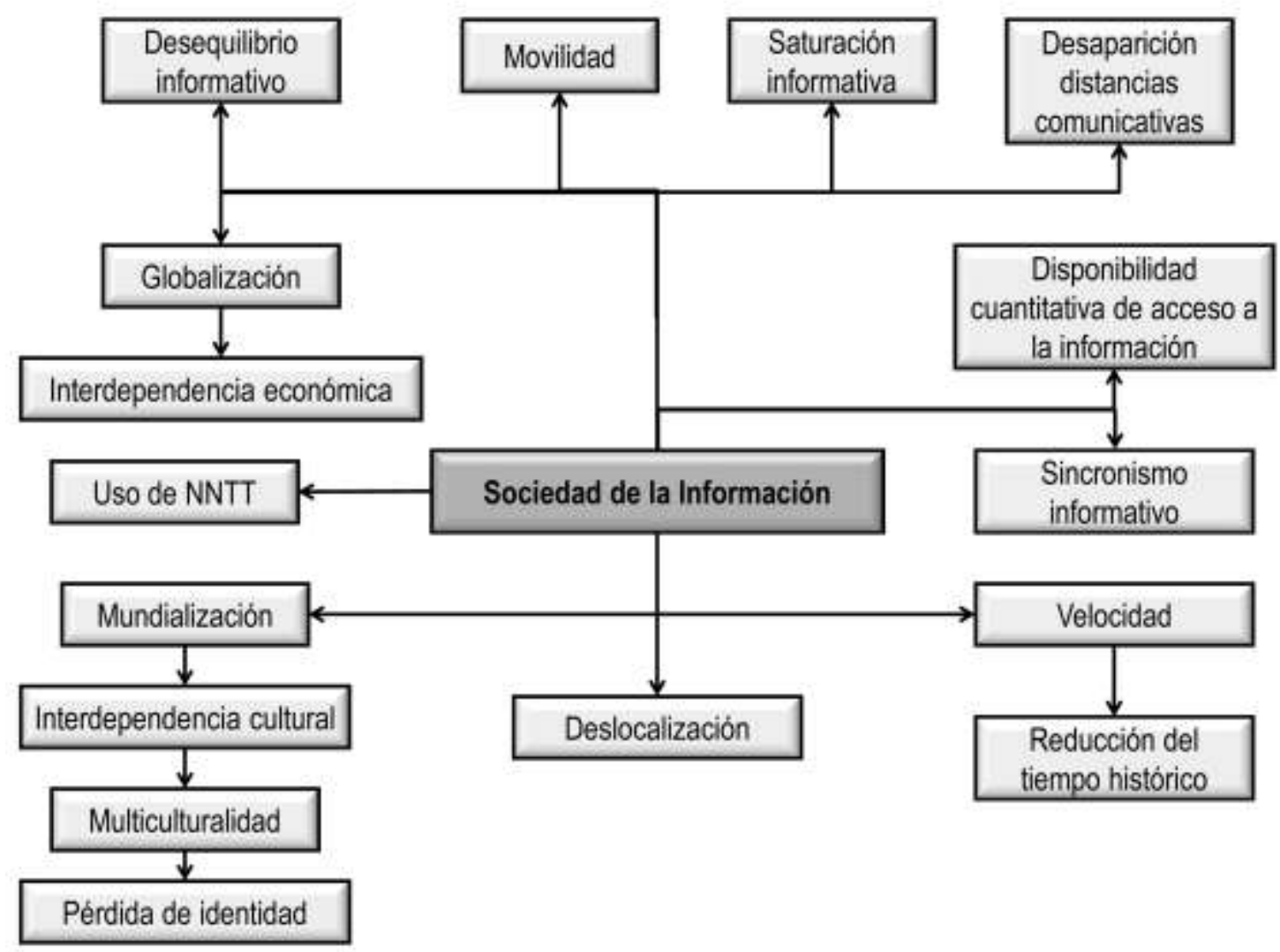

Figura 1. Elementos que configuran la Sociedad de la Información.

Fuente: Martínez Sánchez (2007: 5).

La figura 1 muestra estos diversos elementos que determinan la Sociedad de la Información, la cual da lugar a tres efectos simultáneos: la mundialización que provoca la interdependencia cultural induciendo a los procesos de multiculturalidad y, por tanto, a la pérdida de identidad cultural; la deslocalización; y el aumento en la velocidad de la información, siendo esta última la que compromete la reducción del tiempo histórico.

Por otro lado, la Sociedad de la Información promueve el uso de la tecnología; la disponibilidad cuantitativa de acceso a la información que 
influye en la correspondencia temporal de las noticias o sincronismo informativo; la desaparición de las distancias comunicativas; el desequilibrio; y la saturación de informaciones.

En resumen, la Sociedad de la Información se identifica por la alteración en los ámbitos económico, político y de organización social cuyo elemento distintivo es el empleo de los medios tecnológicos y digitales; los mismos, como subrayan Coll y Monereo (2008: 19), "comportan nuevas maneras de trabajar, de comunicarnos, de relacionarnos, de aprender, de pensar $y$, en suma, de vivir".

Sin embargo, la Sociedad de la Información por sí misma no provocaría este cambio en las formas sociales (Castells, 2001) y, por tanto, se hace obligado educar y capacitar a los ciudadanos (menores, jóvenes y adultos) de forma eficiente para afrontar esta concentración ingente de información.

Este escenario implica una nueva perspectiva en los sistemas educativos, correspondiendo a los mismos capacitar en la adquisición de habilidades y destrezas para gestionar la información y los nuevos conocimientos, donde la generación de estos últimos partirá de las experiencias previas; seguido de una búsqueda de información basada en las necesidades del individuo; $y$, cuyo último avance será una selección y organización de los datos que provocará el proceso cognitivo.

"En las sociedades del conocimiento todos tendremos que aprender a desenvolvernos con soltura en medio de la avalancha aplastante de informaciones, y también a desarrollar el espíritu crítico y las capacidades cognitivas suficientes para diferenciar la información 'útil' de la que no lo es" (UNESCO, 2005: 20).

Cabero (2008:20) indica "la realidad es que toda tecnología no sólo transfiere información, sino que al mismo tiempo está transmitiendo valores y actitudes, algunas veces incluso no perceptibles por las personas", de esta forma las TIC se transmutan en elementos inapreciables, de forma concreta para la educación y de manera general para las sociedades, en el desarrollo 
de una ciudadanía competente que progresa hacia formas de vida sostenibles, minorando los efectos más perjudiciales de la globalización, las desigualdades.

En definitiva, la incorporación de los recursos tecnológicos en los centros educativos promueve nuevas formas de generación del conocimiento y, por otro lado, valores y actitudes que mejoren la sociedad globalizada en la que nos encontramos inmersos, donde el papel del educador social cobra un mayor protagonismo.

\section{El binomio TIC - educación social}

La praxis de la ocupación profesional promovida por la Educación Social es fundamentalmente la socialización de los individuos, promoviendo actuaciones encaminadas a potenciar la incorporación de las personas a la sociedad de forma dinámica y competente; este hecho infiere la conciencia de estos profesionales, dedicados a este campo, de los aspectos que intervienen en la obtención de esta finalidad; entre los mismos influye, en estos momentos, la incorporación de las TIC, como indica Cabero (2010:39), "sin lugar a dudas en los momentos actuales la penetración de las TIC está alcanzado a todos los sectores de nuestra sociedad, desde la cultura y el ocio, hasta la industria y las instituciones de formación; y por otra, que ya nadie los contemplan como un elemento de añadido al sistema educativo, sino como unos medios significativos para el aprendizaje, entornos de innovación escolar, y para la comunicación e interacción social".

Los individuos deben integrar a sus acciones habituales la tecnología, familiarizándose y empleando la misma, como consecuencia lograrán comprender su naturaleza y las potencialidades que brindan; esta meta, en Europa, se conforma como una de las siete iniciativas que propone la Comisión Europea (2010) en las Estrategias para un crecimiento inteligente, sostenible e integrador para el 2020, en estas actuaciones se promueve la adquisición y el desarrollo de la competencia digital en toda la ciudadanía, lo 
cual como apunta Ferrari (2012: 3) "es un derecho y un deber de los ciudadanos para que puedan ser funcionales en las sociedades actuales".

Por tanto, los sistemas educativos se hallan ante un reto y una obligación, donde el educador social juega un papel relevante; no obstante este desafío subyace, como señala Salinas (2012: 15) "en una nueva forma de entender el aprendizaje a lo largo de la vida, a lo largo del trabajo, y con los otros (en un mundo digital)".

La incorporación del Educador Social al sistema educativo, como una de las alternativas profesionales de las que goza, beneficia estos requerimientos que se le solicita a la educación, atendiendo a la formación y la capacitación de este profesional para efectuar su labor de socialización en los entornos educativos, posibilitando una triple vertiente, en sus actuaciones socioeducativas: hacia el alumnado al que las dirige, hacia los organismos públicos y privados con los que colabora y hacia el resto del equipo con el que coopera (Caride, 2008); las mismas se verán favorecidas con la incorporación digital.

En cuanto a la actuación fundamental del Educador Social, no sólo la adquisición de la competencia digital se convierte en elemento esencial para la inclusión en la sociedad sino también el desarrollo del alfabetismo tecnológico, de donde surge la brecha digital como un aspecto social (Area y Guarro, 2012); sin olvidar "que el futuro próximo se está caracterizando por un aprendizaje embebido, continuo y basado en el aprendizaje social" (Salinas, 2012: 15), un aprendizaje cooperativo fundamentado en la diversidad, la comunicación, la autonomía, la iniciativa y la interacción entre iguales.

En la actualidad, se concibe una nueva idea sobre la brecha digital, en contraste con la circunstancia basada en el obstáculo que provenía del excesivo coste y cuantía económica que suponía el acceso a equipos o dispositivos y a la conexión a la red (Cabero, 2004); esta reciente concepción se encamina a los usos eficaces y funcionales de lo digital, lo cual "se relaciona con la capacidad de cada individuo para lograr explotar los 
recursos de las TIC y aplicarlos a sus necesidades" (Alva, 2015: 275).

En resumen, la brecha digital dificulta el desarrollo de los principios básicos de la educación al producir exclusión; al mismo tiempo, los principios básicos de la socialización al obstaculizar la interacción y la comunicación, tanto con los iguales como con el resto de la sociedad; transformándose en un aspecto inhibidor del bienestar personal; en consecuencia, debe ser contemplado por el educador social como posible situación de riesgo y marginalidad para el alumnado.

\section{Reflexiones finales}

En este artículo hemos intentado manifestar la relevancia que cobran los profesionales de la Educación Social en la sociedad actual, donde se despliega una necesidad por la comunicación y la interacción entre sujetos, una socialización adecuada y eficaz.

Por otro lado, los medios tecnológicos y digitales potencian esta participación social en todas las esferas del desarrollo humano; obligando a la ciudadanía a nuevos aprendizajes y dominios para su desenvolvimiento efectivo.

Es precisamente, los agentes del ámbito de la Educación Social, quienes deben considerar estos nuevos aliados para facilitar la socialización de los individuos en la sociedad.

Asimismo, estos profesionales propician la conexión entre la realidad social y la educativa, entre las que destaca la incorporación de la tecnología en ambas esferas; concretamente el conocimiento, uso y aplicabilidad de estos recursos digitales se articulan para el educador social en una doble vertiente por un lado como elementos para la gestión y administración de su labor profesional y, por otro, como medios que deben integrarse en los procesos de aprendizaje, de comunicación e interacción del alumnado y de cualquier comunidad.

En conclusión, los agentes dedicados profesionalmente al campo de la 
intervención social no pueden obviar los recursos tecnológicos para el ejercicio de sus funciones; en consecuencia, deben conocer las ventajas y limitaciones que implica su aplicación, así como, la no utilización de los mismos, considerando que esta última opción puede provocar la inadaptación social del individuo en la ciudadanía.

\section{Referencias bibliográficas}

ANECA. (2005). Libro Blanco de Grado en Pedagogía y Educación Social (vol. 2). Madrid: ANECA.

ALVA, A.R. (2015). Los nuevos rostros de la desigualdad en el siglo XXI: la brecha digital. Revista Mexicana de Ciencias Políticas y Sociales, 60(223), 265-286. Recuperado de: http://www.revistas.unam.mx/index.php/rmspys/article/view/45387

AREA, M., y GUARRO, A. (2012). La alfabetización informacional y digital: fundamentos pedagógicos para la enseñanza y el aprendizaje competente. Revista Española de Documentación Científica, $\mathrm{n}^{\circ}$ Monográfico, 46-74. DOI: 10.3989/redc.2012.mono.977.

ASOCIACIÓN ESTATAL DE EDUCACIÓN SOCIAL. (2007). Documentos profesionalizadores. Barcelona: ASEDES.

CABERO, J. (2004). Reflexiones sobre la brecha digital y la educación. En F. Soto y J. Rodríguez. (coord.). Tecnología, educación y diversidad: retos y realidades de la inclusión digital. (23-42). Murcia: Consejería de Educación y Cultura.

CABERO, J. (2008). La formación en la sociedad del conocimiento. Indivisa, 23, $13-48$.

Recuperado

de: http://tecnologiaedu.us.es/cuestionario/bibliovir/jca23.pdf

CABERO, J. (2010). Los retos de la integración de las TIC en los procesos educativos. Límites y posibilidades. Revista Perspectiva Educacional, 49(1), 32-61. Recuperado de: http://tecnologiaedu.us.es/tecnoedu/images/stories/jca73.pdf 
CARIDE, J. A. (2008). El grado en Educación Social en la construcción del espacio europeo de educación superior. Educación XXI, 11, 103-131. Recuperado de: http://www.redalyc.org/pdf/706/70601 106.pdf

CASTELLS, M. (2001). Materiales para una teoría preliminar sobre la sociedad de redes. Revista de Educación, (número extraordinario Globalización y Educación), 41-58. Recuperado de: http://www.mecd.gob.es/revistade-educacion/numeros-revista-educacion/numerosanteriores/2001/re2001.html

CASTELLS, M. (2005). The Network Society: from Knowledge to Policy. En M. Castells y G. Cardoso. The Network Society From Knowledge to Policy. (pp. 3-22). Washington: Center for Transatlantic Relations. Recuperado de:

http://www.umass.edu/digitalcenter/research/pdfs/JF_NetworkSociety.p df

CHAMSEDDINE, M. (2013). Aproximación histórica a una de las profesiones sociales: La educación Social. RES. Revista de Educación Social, 17, 1-13. Recuperado de: http://www.eduso.net/res/pdf/17/aprox res \%2017.pdf

COLL, C. y MONEREO, C. (2008). Educación y aprendizaje en el siglo XXI: Nuevas herramientas, nuevos escenarios, nuevas finalidades. En C. Coll y C. Monereo (coord.). Psicología de la educación virtual. (pp.19-53). Madrid: Morata.

COMISIÓN EUROPEA. (2010). Europa 2020: Una estrategia para un crecimiento inteligente, sostenible e integrador. COM (2010) 2020. Recuperado de: http://eur-

lex.europa.eu/LexUriServ/LexUriServ.do? uri=COM:2010:2020:FIN:ES:PDF.

FERRARI, A. (2012). Digital Competence in practice: An analysis of frameworks. Seville: JRC-IPTS. DOI:10.2791/82116.

GAIRÍN, J. (2004). La organización escolar: contexto y texto de actuación. (4 ${ }^{a}$ ed.). Madrid: La Muralla.

GONZÁLEZ, J., y WAGENAAR, R. (2003). Tuning Educational Structures in Europe. 
Informe final. Fase 1. Bilbao: Universidad de Deusto.

MARTíN, A. H., LÓPEZ, E., y GONZÁLEZ, J. (2013). Reflexiones sobre la Sociedad de la Información y las Tecnologías de la Información y la Comunicación. En E. Corbi, E. López Meneses, F. M. Sirignano, J. L. Sarasola y J. González (Dir.). II Seminario científico Internacional sobre Formación Didáctica con Tecnologías Web 2.0. (pp. 1-7). Sevilla: AFOE.

MARTÍNEZ SÁNCHEZ, F. (2007). La sociedad de la información. En J. Cabero (coord.). Tecnología educativa. (pp.1-12). Madrid: McGraw-Hill.

PÉREZ SERRANO, G. (2005). Presentación Monográfico Educación Social. Revista de Educación, 335. 7-18. Recuperado de: http://www.revistaeducacion.mec.es/re336/re336.pdf

REAL ACADEMIA ESPAÑOLA. (2001). Educación. En Diccionario de la lengua española (22. ${ }^{a}$ ed.). Recuperado de: http://lema.rae.es/drae/?val=educacion

REAL ACADEMIA ESPAÑOLA. (2001). Social. En Diccionario de la lengua española (22. ${ }^{a}$ ed.). Recuperado de: http://lema.rae.es/drae/? val=social RESOLUCIÓN de 5 de junio de 2007, de la Dirección General de Trabajo, por la que se registra y publica del I Convenio colectivo marco estatal de acción e intervención social, publicado en el BOE núm. 146 de 19 de junio de 2007.

SALINAS, J. (2012). La investigación ante los desafíos de los escenarios de aprendizaje futuros. RED, Revista de Educación a Distancia, 32. 1-23. Recuperado de: http://www.um.es/ead/red/32/salinas.pdf UNESCO. (2005). Hacia las sociedades del conocimiento. Paris: UNESCO.

\section{Cómo citar este artículo:}

Sampedro Requena B.E. (2016). Las TIC y la educación social en el siglo XXI. EDMETIC, Revista de Educación Mediática y TIC, 5(1), 8-24. 